\title{
An Efficient Orthogonal Frequency Division Multiplexing (OFDM) System and Performance Analysis of Digital Audio Broadcasting (DAB) System
}

\author{
Drakshayini M. N. \\ VTU \\ Associate Professor, \\ Dept. OF ECE, TJIT, Bangalore
}

\author{
Arun Vikas Singh, PhD \\ VTU \\ Professor and Head \\ Dept. OF ECE, TJIT, Bangalore
}

\begin{abstract}
The foremost objective of digital modulation today is to provide high spectral efficiency. OFDM is a multicarrier digital modulation technique which offers high spectral efficiency. In this paper an efficient OFDM system under AWGN channel is designed using MATLAB and performance analysis is done for the system by evaluating BER and SNR. Frequency Modulation (FM) mainly suffers from Multipath fading and ISI. FM is suitable for fixed reception but it is not suitable for mobile reception since it suffers loss of broadcasting quality. These issues are overcome in DAB. DAB is a digital radio or high definition radio which offers high audio quality. This paper also proposes that designing DAB using OFDM under Rayleigh fading channel. Performance analysis of DAB system is done for the audio input by evaluating BER and SNR.
\end{abstract}

\section{General Terms}

Symbol Mapping, Pilot insertion, Convolutional Encoding and Puncturing and Time Interleaving

\section{Keywords}

OFDM, DAB, ISI, BER, SNR.

\section{INTRODUCTION}

Recent year's Wireless communication requires high spectral efficiency and high robustness. OFDM is a method of multi carrier digital modulation technique which provides high spectral efficiency and high robustness [1]. OFDM is used in many applications such as digital video broadcasting, digital audio broadcasting, ADSL, LTE, Wireless LAN standards and Digital radio mondiale etc [2-3]. This paper describes to simulate an efficient OFDM system. The idea of this development is to study and understand the structure of an OFDM system. OFDM system is developed using MATLAB programming and simulation results are observed at each stage, for the given input bit stream output is obtained with minimal bit error rate.

Although FM has many advantages over AM, FM mainly suffers from Multipath fading and ISI. FM is suitable for immobile reception but it experiences loss of broadcasting quality during mobile reception. These issues are resolved in Digital Audio Broadcasting (DAB). DAB is digital radio or high definition radio which broadcasts radio services from studio to receiver. DAB is aimed to offer high quality digital audio programs and data services to fixed, mobile and handy devices. It was established in 1990s by Eureka 147 DAB

Project [4]. This paper describes the designing of an OFDM system under AWGN channel and DAB system using OFDM under Rayleigh fading channel. Performance analysis of an OFDM system and DAB system are done by evaluating BER and SNR.

\section{OVERVIEW OF AN OFDM}

OFDM is a special case of multi carrier digital modulation technique in which a single data stream will be transmitted over a many narrowband closely spaced subcarriers and maintains orthogonality among subcarriers. OFDM technique may be used as either modulation or multiplexing or both. One of the main reason to use OFDM is, it offers high spectral efficiency and provides high robustness against multipath fading and narrowband interference [5]. Mathematically [6] OFDM signal can be described as in equation (1).

$$
c(t)=\sum_{n=0}^{N-1} S_{n}(t) \sin \left(2 \pi f_{n} t\right)
$$

where $S_{n}(t)=$ symbol mapped to select constellation, $N=$ FFT length, $f_{n}=$ orthogonal frequency.

In OFDM the subcarriers are chosen in such a way that they are mutually orthogonal and which eliminates the cross talk between the subcarriers [7]. Equation (2) defines the orthogonality of two signals. Orthogonality of two signals can be defined as the signals they are linearly independent and their dot product is zero.

$$
\int_{x}^{y} X_{p}(t) X_{q}^{*}(t) d t=\left\{\begin{array}{l}
K \text { for } p=q \\
0 \text { for } p \neq q
\end{array}\right.
$$

where $[\mathrm{x}, \mathrm{y}]$ is for one symbol period.

Figure 1 illustrates the block diagram of the OFDM systems [8]. Stage I symbol mapping does one to one mapping, this stage takes binary data stream input and each bit is represented as complex valued number on the constellation. In stage II serial to parallel converter transforms serial symbol sequence into parallel sequence.

In Stage III, pilot carriers are added into the OFDM Symbol. Pilot carriers are non-information carrier and it does not carry any information. Pilot carriers are the complex valued number represented on the constellation. The pilot carriers are used for channel estimation. Stage IV, the Inverse Fast Fourier Transform (IFFT) stage. IFFT is an efficient method used for generating an OFDM symbol. IFFT transforms frequency domain symbols into time domain waveform and produces the combined set of subcarriers which are multiplexed and maintains orthogonality between the sub-carriers. 


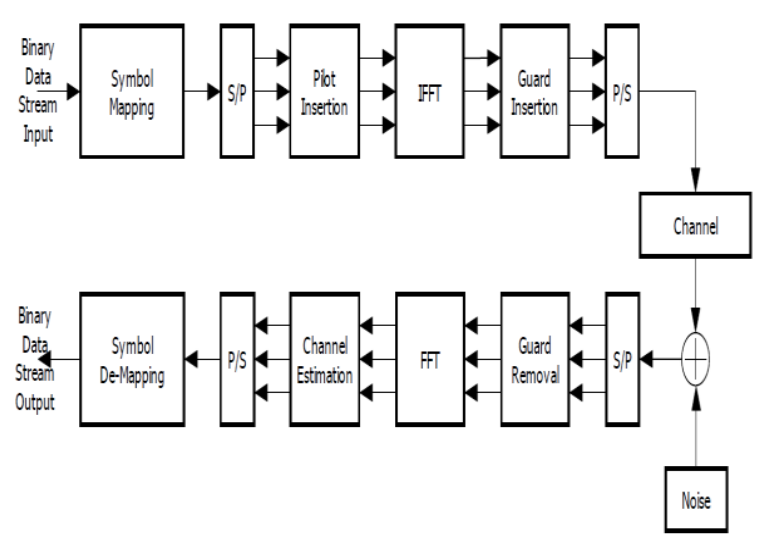

Figure 1: Block diagram of typical Orthogonal Frequency Division Multiplexing System.

Stage V is the guard interval insertion, which affixes a cyclic prefix to the beginning of every OFDM symbols to diminish the effect of ISI and ICI [9]. Figure 2 indicates the OFDM symbol with cyclic prefix.

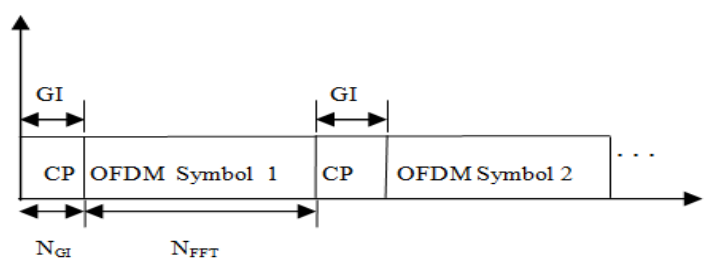

Figure 2: Representation of a sequence of Orthogonal frequency division multiplexing symbols with cyclic prefix.

In Stage VI the parallel to serial converter converts the OFDM Symbols into serial sequence which is the real-valued base band OFDM waveform.

At the receiver part of figure 1, serial to parallel converter converts OFDM waveforms into parallel sequence and guard removal stage will eliminates the appended cyclic prefix from the OFDM symbol. FFT stage transforms time domain sequence into a frequency domain sequence and performs demultiplexing. Channel estimation stage with the help of pilot carriers finds beginning of the OFDM symbol. Parallel to serial converter stage converts parallel data sequence into a serial sequence. Symbol de-mapping transforms the complex valued number into binary data stream considering their phase and amplitude values.

\section{OVERVIEW OF DAB}

The novel digital radio system DAB may be defined as Digital Radio or high definition radio is an innovative and universal multimedia broadcasting system. It has replaced the present $\mathrm{AM}$ and FM audio broadcasting services in many parts of the world. DAB is most suitable for mobile reception and provides high robustness against multipath reception. DAB utilizes single frequency networks (SFNs) to attain high frequency efficiency.

DAB provides high quality digital audio services as mono, two-channel or multichannel stereophonic. DAB can convey program-related data and a multiplex of data services such as travel and traffic information, static and moving pictures, etc. DAB works quite different when we compare to conventional broadcasting systems. DAB consists of new system components for performing perceptual audio coding, channel coding, modulation and multiplex management and data transmission protocols [10].

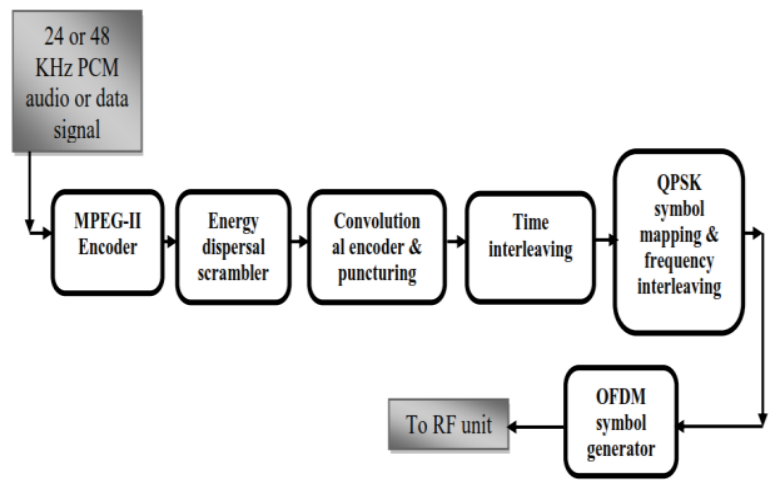

Figure 3: DAB transmitter block diagram

Figure 3 displays the transmitter block diagram of the DAB system. The overall DAB transmission can be distributed into the number of functional blocks and these blocks process the input signal and produces the complete DAB signal. The analog signal i.e. audio signal or data signal is given as input to MPEG layer-2 encoder which generates the encoded data. In order to make sure that the appropriate energy dispersal in the transmitted signal, distinct inputs of the energy dispersal scramblers presented in Figure 3 is to be scrambled with the help of modulo- 2 addition and PRBS [11]. The scrambled bit stream is then given to punctured convolutional encoder to perform forward error correction and convolutional encoding. The coded bit-stream are rearranged using time interleaving. Time interleaving is done basically to increase the robustness of the transmitted data. Time interleaved data is then subjected to QPSK modulation this block takes binary data stream input and each bit is represented as complex valued number on the constellation and does one to one mapping. After the QPSK modulation symbols are given to OFDM block where final DAB transmission signals are generated.

\section{PERFORMANCE ANALYSIS 4.1 OFDM system using MATLAB Simulation}

Figures 1 illustrate the block diagram of OFDM system in which we have simulated using MATLAB. The objective of this modeling and simulation is to perform the performance analysis of OFDM system. Performance analysis is done by evaluating the BER and SNR of the OFDM system. The parameter used for designing an OFDM system are listed in Table 1.

Table 1: OFDM parameters

\begin{tabular}{|c|c|}
\hline Parameter & Value \\
\hline FFT Size & 64 \\
\hline Number of subcarriers used & 4 \\
\hline Modulation type & QPSK \\
\hline One OFDM Symbol length & 16 \\
\hline Cyclic prefix length & $\begin{array}{c}10 \% \text { of One } \\
\text { OFDM Symbol }\end{array}$ \\
\hline
\end{tabular}

In this design a set of binary stream is given as input across the transmitter of OFDM and frame based processing is employed and the type of modulation used is QPSK 
modulation. The efficient IFFT algorithm is used for subcarrier modulation and multiplexing. To overcome ISI cyclic prefix is affixed at the beginning of each OFDM symbol. The length of guard interval chosen is $10 \%$ of One OFDM Symbol. AWGN channel is used for the analysis. At receiver block received signal is processed to remove noise, received signal from channel is converted into digital data. This binary stream is partition into vectors and the appended cyclic prefix is removed from the OFDM symbol. By performing FFT on each symbol, vectors have been extracted and calculated the phase of each symbol. Then calculated the phase difference between one symbol period to the next, finally each symbol phase will be decoded as binary data. Performance analysis done by calculating BER and SNR. The proposed design achieves to obtain improved SNR and lower BER.

\subsection{DAB system using MATLAB Simulation}

Figure 4 illustrates the block diagram of the DAB system.

DAB system is modeled and simulated using MATLAB programming. The aim of this simulation is to evaluate the BER and SNR of the DAB system using convolutional coding with puncturing method. Puncturing method lets the encoding and decoding of higher rate codes using standard rate 1/2 encoders and decoders. The simulation parameters for DAB mode II are listed in Table 2. Frame based processing is performed in this simulation model under Rayleigh fading channel for the performance analysis. DAB frame structure is formed by one synchronization channel is utilized for transmission frame synchronization and transmitter identification, three Fast Information Channel [FIC] used for quick access of information in the receiver and 72 Main Service Channel [MSC] used to convey audio and data services.

Table 2: Mode II Parameters of DAB system

\begin{tabular}{|c|c|}
\hline Parameter & Value \\
\hline Number of subcarriers & 384 \\
\hline Subcarrier spacing & $4 \mathrm{kHz}$ \\
\hline Transmission Frame duration & $24 \mathrm{~ms}$ \\
\hline Symbol duration & $321 \mu \mathrm{s}$ \\
\hline Guard Interval & $62 \mu \mathrm{s}$ \\
\hline Null Symbol duration & $324 \mu \mathrm{s}$ \\
\hline OFDM symbols per transmission frame & 76 \\
\hline OFDM symbols with PR data & 1 \\
\hline OFDM symbols with FIC data & 3 \\
\hline OFDM symbols with MSC data & 72 \\
\hline
\end{tabular}

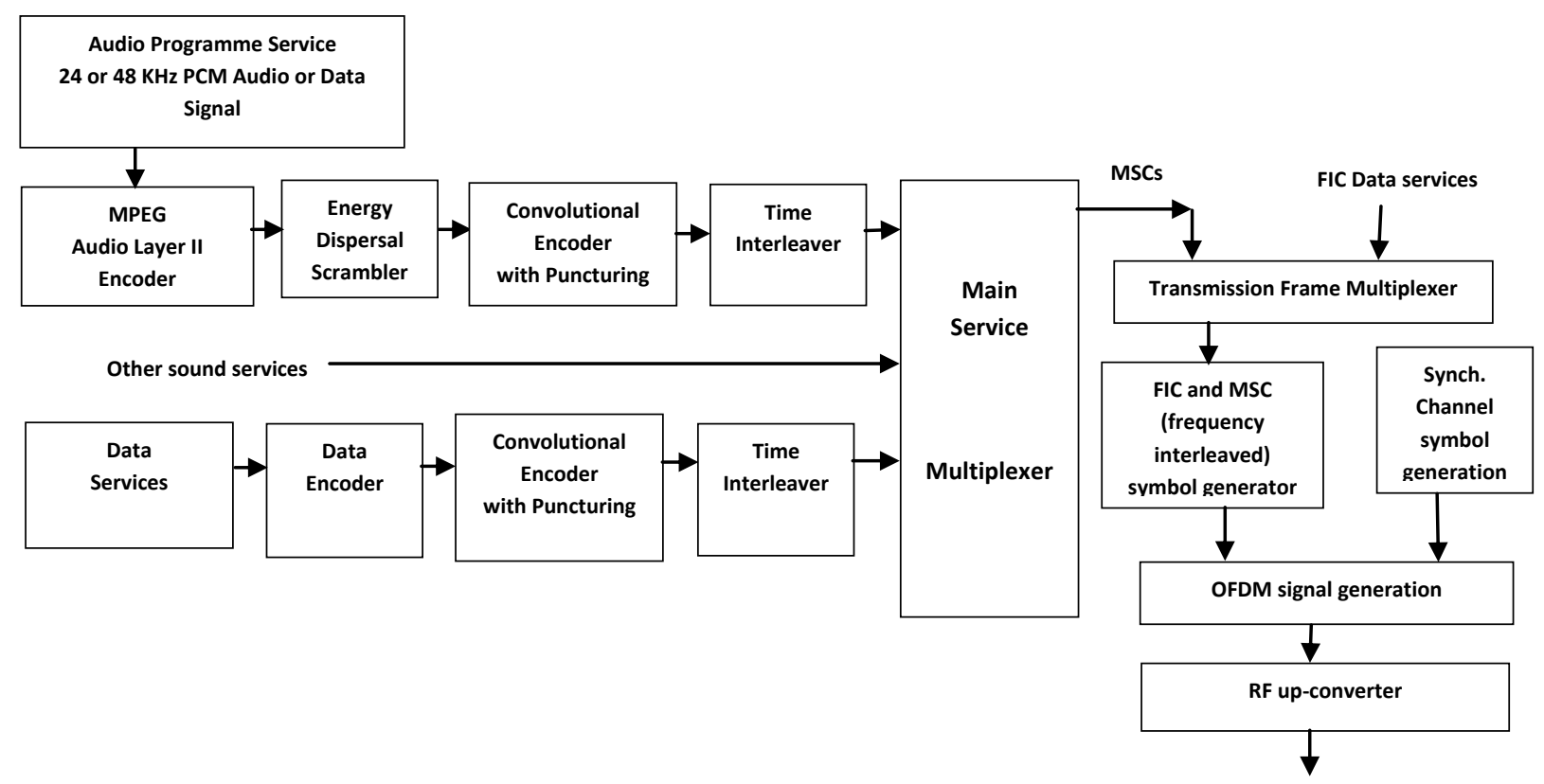

Figure 4: Block diagram of the DAB system DAB Transmission Signals

\section{SIMULATION RESULTS 5.1 OFDM system}

This section describes the simulation results of OFDM system under AWGN channel. A binary stream of data input is given to the OFDM system which is shown in figure 5. Figure 6 represents the OFDM signal in time domain. Figure 7 illustrates the Sub-carriers carrying OFDM symbols. Figure 8 and figure 9 signifies the IFFT of the subcarriers and FFT of the subcarriers respectively. Figure 10 represents the received data with fewer errors. Based on the transmitted and received data bit error rate and SNR is calculated. Figure 11 illustrates the BER versus SNR plot for OFDM system under AWGN channel. This plot shows that as SNR increases BER decreases. Simulation result shows improved SNR of $11 \mathrm{~dB}$ and reduced BER of $10^{-0.1}$.

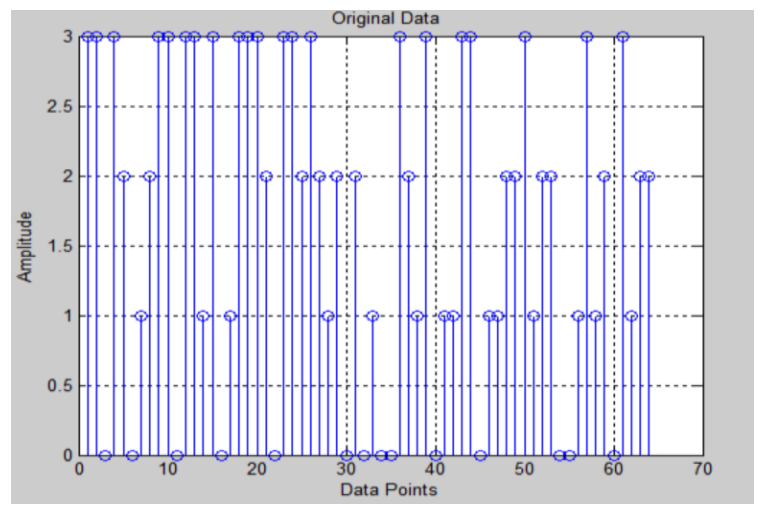

Figure 5: Input Data 


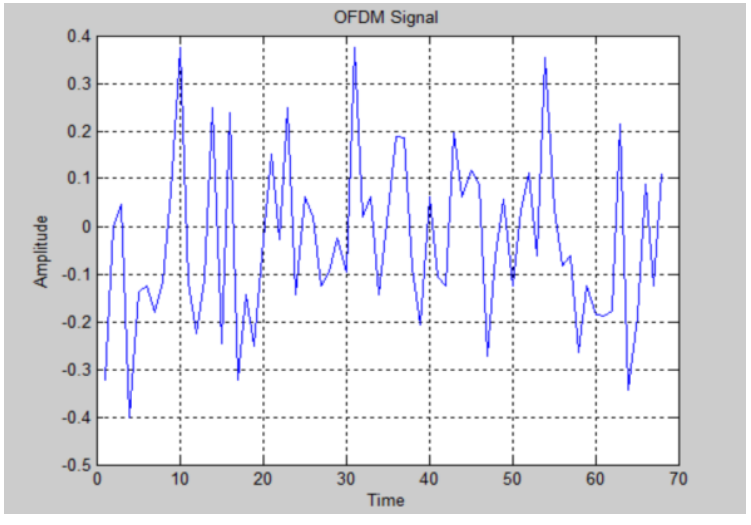

Figure 6: OFDM Signal in time domain

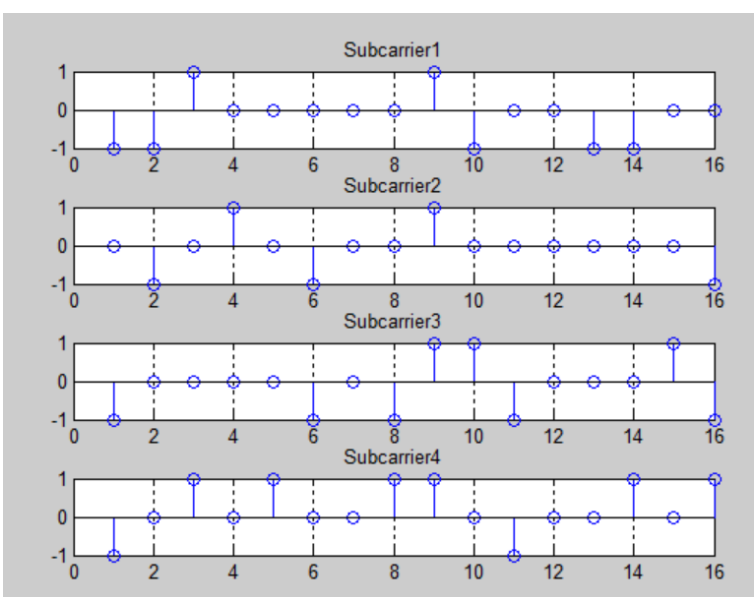

Figure 7: Subcarriers carrying OFDM symbols

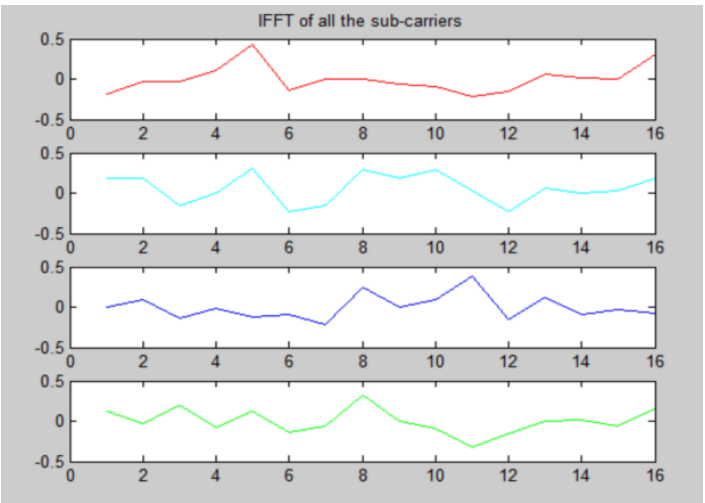

Figure 8: IFFT of the subcarriers

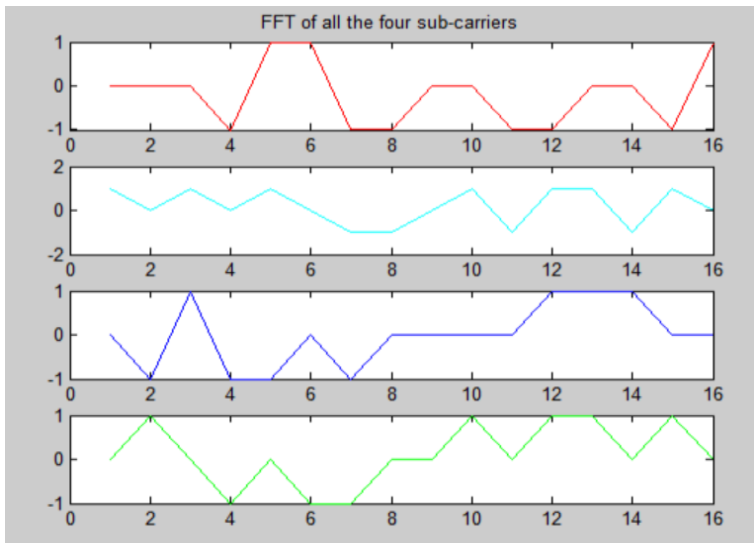

Figure 9: FFT of the subcarriers

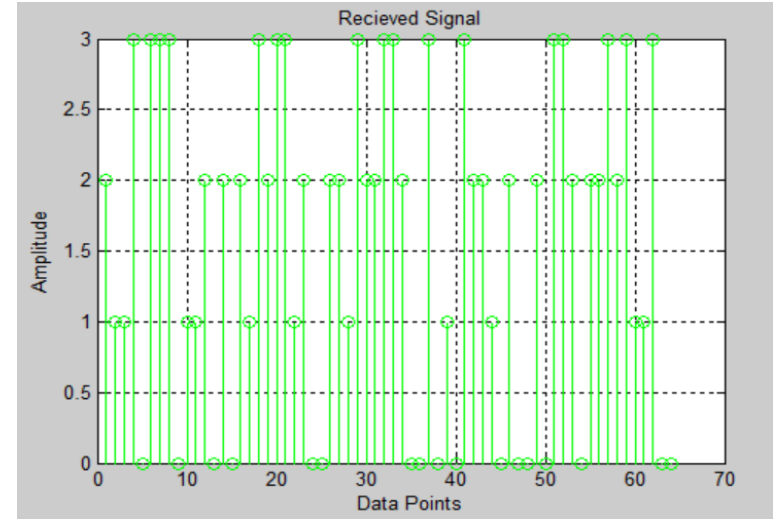

Figure 10: Received data

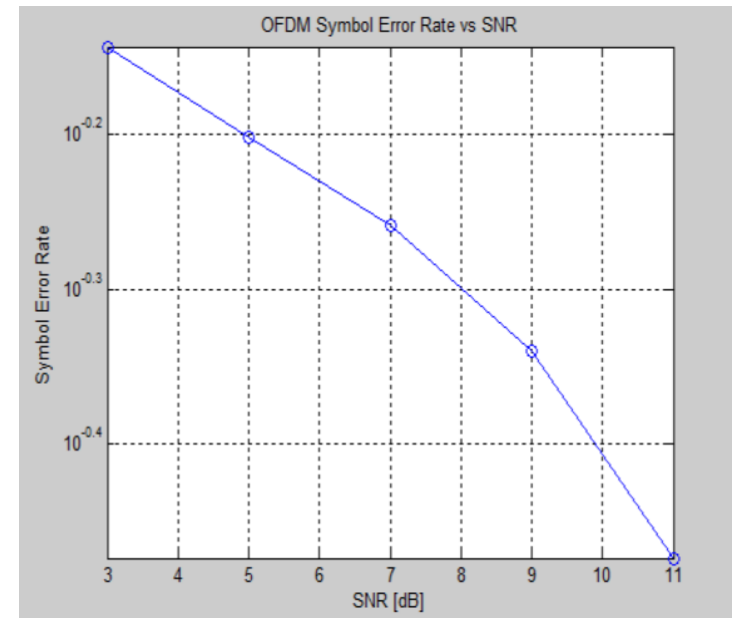

Figure 11: BER vs. SNR for OFDM system under AWGN channel

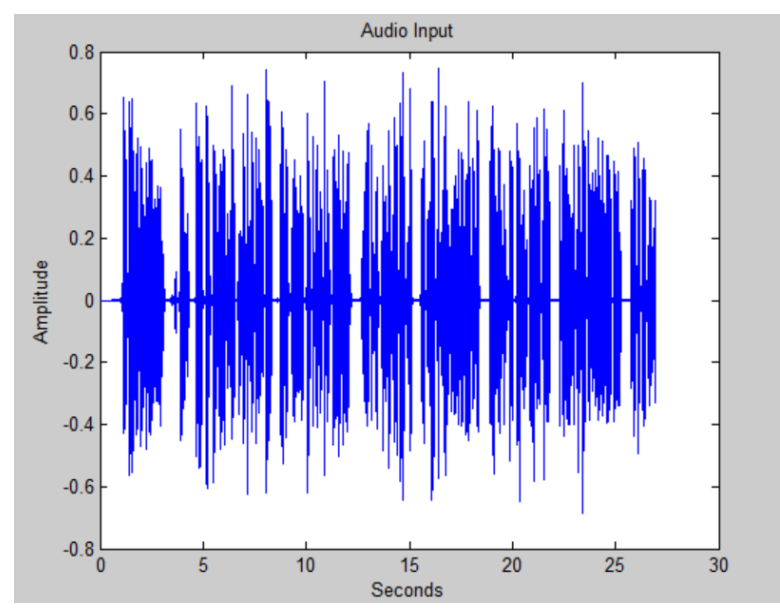

Figure 12: Audio input to DAB system

\subsection{DAB system}

This section illustrates the simulated results of DAB system for Rayleigh fading channel. An audio input is given to the DAB system which is shown in figure 12. An audio signal is processed and transmitted under Rayleigh fading channel. Figure 13 illustrates BER vs. SNR for DAB system. This plot shows that as SNR increases BER decreases. Simulation result shows improved SNR of $16 \mathrm{~dB}$ and BER of $10^{-0.9}$. 


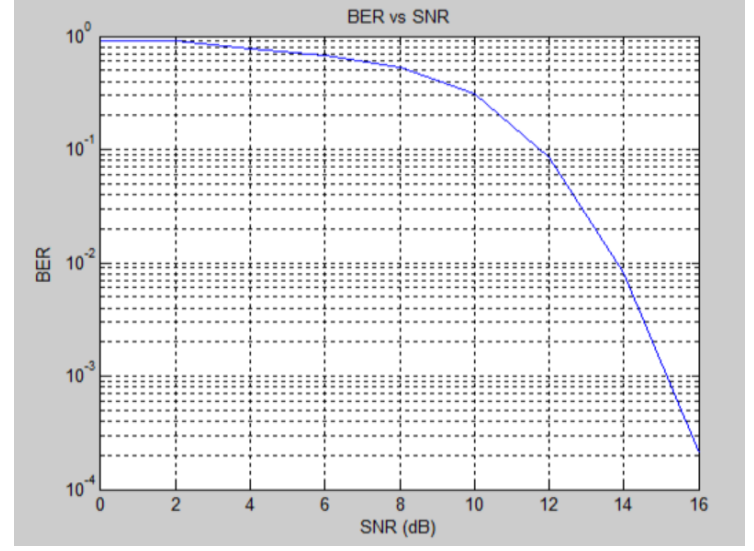

Figure 13: BER vs. SNR for DAB system under Rayleigh fading channel

\section{CONCLUSION}

A simulation based performance analysis of an efficient OFDM system and DAB system by using convolutional coding and puncturing technique is presented in this paper. The proposed technique provides the improved BER performance for both OFDM system and DAB system. Many applications use OFDM system because of its high spectral efficiency. Based on simulations, DAB system seems to be appropriate radio broadcasting technology to offer high performance in diverse transmission channels. Based on the present trend in wireless technologies, DAB will turn out to be predominant in future radio broadcasting.

\section{REFERENCES}

[1] Deepak Sharma and Praveen Srivastava "OFDM Simulator Using MATLAB" International Journal of Emerging Technology and Advanced Engineering com, ISSN 2250-2459, ISO 9001:2008 Certified Journal, Volume 3, Issue 9, September 2013.

[2] Rachana Khanduri \& Dehradun S. S. Rattan "Performance Comparison Analysis between IEEE $802.11 \mathrm{a} / \mathrm{b} / \mathrm{g} / \mathrm{n}$ Standards" International Journal of Computer Applications (0975 - 8887) Volume78- No.1, September 2013

[3] D. Perez-Calderon, V. Baena-Lecuyer, J. Chavez, A. C. Oria and J. G. Doblado, "Simplified Detection for DVBNGH MIMO Decoders," in IEEE Transactions on
Broadcasting, vol. 61, no. 1, pp. 84-90, March 2015. doi: 10.1109/TBC.2014.2367991

[4] V.B. Malode and Anuja D. Saravade " Performance prediction of DAB modulation and transmission using Rayleigh Fading channel" International Journal of Emerging Trends \& Technology in Computer Science, ISSN 2278-6856 Volume 3, Issue 4 July-August 2014

[5] "OFDM for Wireless Communication System" Ramjee Prasad, Artech house, Inc. Boston London, ISBN 158053-796-0.

[6] IEEE 802.11 specification - "Orthogonal frequency division multiplexing (OFDM) PHY specification for the $5 \mathrm{GHz}$ band"- Chapter 17.

[7] N. LaSorte, W. J. Barnes and H. H. Refai, "The History of Orthogonal Frequency Division Multiplexing," Global Telecommunications Conference, 2008. IEEE GLOBECOM 2008. IEEE, New Orleans, LO, 2008, pp. 1-5.doi: 10.1109/GLOCOM.2008.ECP.690

[8] Keith E. Nolan, Departments of Electronics and Electrical Engineering, University of Dublin, Trinity College, Dublin 2, "Reconfigurable OFDM systems " May 2005.

[9] Jibin Wang and Jinkang Zhu, "A Novel Time and Frequency Synchronization Approach for OFDM Systems," Information Technology and Applications, 2005. ICITA 2005. Third International Conference on, Sydney, NSW, 2005, pp. 311-315.doi 10.1109/ICITA.2005.38

[10] "Digital Audio Broadcasting Principles and Applications of Digital Radio" Second Edition Edited by WOLFGANG HOEG Berlin, Germany and THOMAS LAUTERBACH University of Applied Sciences, Nuernberg, Germany, Copyright 2003 John Wiley \& Sons Ltd, ISBN 0-470-85013-2

[11] ETSI., "Radio Broadcasting Systems; Digital Audio Broadcasting (DAB) to mobile,portable and fixed receivers," EN 300 401, V1.3.3, (2001-05), April 2001.Sannella, M. J. 1994 Constraint Satisfaction and Debugging for Interactive User Interfaces. Doctoral Thesis. UMI Order Number: UMI Order No. GAX9509398., University of Washington. 